\title{
Searching for Saddle Points of Potential Energy Surfaces by Following a Reduced Gradient
}

\author{
WOLFGANG QUAPP, ${ }^{1}$ MICHAEL HIRSCH, ${ }^{1}$ OLAF IMIG, ${ }^{2}$ \\ DIETMAR HEIDRICH ${ }^{2}$ \\ ${ }^{1}$ Mathematisches Institut, Universität Leipzig, Augustus-Platz, D-04109 Leipzig, Germany \\ ${ }^{2}$ Institut für Physikalische und Theoretische Chemie, Universität Leipzig, Augustus-Platz, D-04109 \\ Leipzig, Germany
}

Received 8 December 1997; accepted 16 February 1998

\begin{abstract}
The old coordinate driving procedure to find transition structures in chemical systems is revisited. The well-known gradient criterion, $\nabla E(\mathbf{x})=\mathbf{0}$, which defines the stationary points of the potential energy surface (PES), is reduced by one equation corresponding to one search direction. In this manner, abstract curves can be defined connecting stationary points of the PES. Starting at a given minimum, one follows a well-selected coordinate to reach the saddle of interest. Usually, but not necessarily, this coordinate will be related to the reaction progress. The method, called reduced gradient following (RGF), locally has an explicit analytical definition. We present a predictor-corrector method for tracing such curves. RGF uses the gradient and the Hessian matrix or updates of the latter at every curve point. For the purpose of testing a whole surface, the six-dimensional PES of formaldehyde, $\mathrm{H}_{2} \mathrm{CO}$, was explored by RGF using the restricted Hartree-Fock (RHF) method and the STO-3G basis set. Forty-nine minima and saddle points of different indices were found. At least seven stationary points representing bonded structures were detected in addition to those located using another search algorithm on the same level of theory. Further examples are the localization of the saddle for the $\mathrm{HCN} \rightleftharpoons \mathrm{CNH}$ isomerization (used for steplength tests) and for the ring closure of azidoazomethine to $1 \mathrm{H}$-tetrazole. The results show that following the reduced gradient may represent a serious alternative to other methods used to locate saddle points in quantum chemistry. (c) 1998 John Wiley \& Sons, Inc. J Comput Chem 19: 1087-1100, 1998
\end{abstract}

Keywords: saddle point; distinguished coordinate; valley-ridge inflection point; $\mathrm{H}_{2} \mathrm{CO}$ potential energy surface; $\mathrm{HCN} \rightleftharpoons \mathrm{CNH}$ isomerization; azidoazomethine $\rightleftharpoons 1 H$-tetrazole isomerization

Correspondence to: W. Quapp; e-mail: quapp@server1.rz.unileipzig.de

Contract/grant sponsor: Deutsche Forschungsgemeinschaft 


\section{Introduction}

$\mathbf{T}$ he concept of the potential energy surface (PES) forms the basis upon which most reaction theories are defined. ${ }^{1,2}$ Chemical reactions are governed by the PES of the molecule(s) involved. The chemically most important features of the PES are the reactant and product minimum (Min), and a saddle point (SP) lying somewhere between the minima. This SP of index 1 forms the transition structure of conventional transition state theory. ${ }^{3}$ All so-called stationary points (StP) of the PES are characterized by the condition:

$$
\nabla E(\mathbf{x})=\mathbf{0},
$$

where $E(\mathbf{x})$ is the function of the PES, and $\nabla E(\mathbf{x})$ is its gradient vector in the configuration space, $\mathbf{R}^{N}$, defined by the coordinates $\mathbf{x}$ of the molecule where $N=3 n$ ( $n=$ number of atoms) if Cartesian coordinates are used, or $N=3 n-6$ for internal coordinates.

If a saddle point of index 1 is known, the steepest descent paths ${ }^{4}$ in both directions of the eigenvector along the decay direction may be defined. The eigenvector is associated with the negative eigenvalue of the Hessian matrix (the second derivatives of the PES). The combination of these descents from the SP to the two minima is frequently termed the reaction path. ${ }^{1,2,4-6}$ However, saddles are considerably more difficult to locate than the reactant or product minimum, and algorithms to locate the SP are still the subject of intensive theoretical effort (see ref. 7, among others). This is because of the different character of the Hessian of these two kinds of extrema, SP and minima, of the PES. At a minimum, all eigenvalues are positive. At a SP of index 1 there is one negative eigenvalue. The resulting mixture of eigenvalues causes a divergence of steepest descent lines in the neighborhood of a SP. Different algorithms to locate saddles are proposed and used in standard quantum chemistry program packages (cf. GAMESS-UK ${ }^{8}$ ).

In this study, we use a simple idea, which transforms the old "distinguished coordinate method" $^{\prime \prime, 10}$ into a new mathematical form. Eq. (1) is valid at extrema of the PES. But single components of the gradient can also vanish in the neighborhood of an extremum, as well as in other regions of the PES. We will use this property. A curve of points $\mathbf{x}$ is followed which fulfills the
$N-1$ equations:

$$
\frac{\partial E(\mathbf{x})}{\partial x^{i}}=0, \quad i=1, \ldots, \ldots, \ldots, N
$$

omitting the $k$ th equation. ${ }^{10}$ This gives the $(N-1)$-dimensional zero vector of the "reduced gradient"; the method is subsequently called reduced gradient following (RGF). The idea of the method may be explained using the surface shown
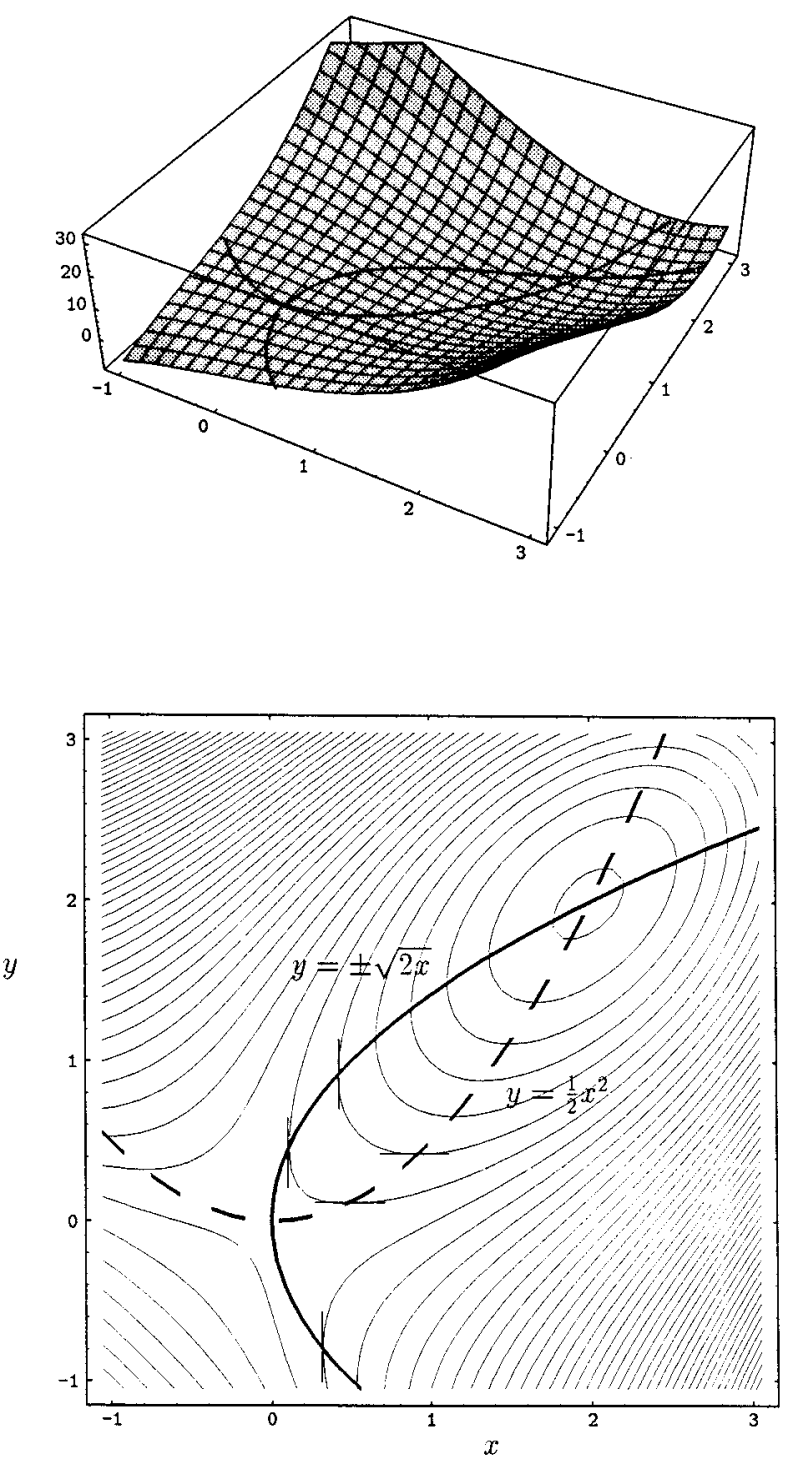

FIGURE 1. Two-dimensional model potential surface $E(x, y)=x^{3}+y^{3}-6 x y$ with the two bold-faced RGF curves $E_{x}=0$ (dashed) and $E_{y}=0$. These curves connect the minimum of the surface with the saddle point. Their intersection locates the two stationary points of the surface. 
in Figure 1, and:

$$
E(x, y)=x^{3}+y^{3}-6 x y
$$

where $x=x_{1}$ and $y=x_{2}$. It has the two stationary points, $\mathrm{Min}=(2,2)$, and $\mathrm{SP}=(0,0)$. The RGF equations become:

$$
\begin{gathered}
E_{x}(x, y)=3 x^{2}-6 y=0 \text { or } \\
E_{y}(x, y)=3 y^{2}-6 x=0
\end{gathered}
$$

with the two curves $y=\frac{1}{2} x^{2}$ or $y= \pm \sqrt{2 x}$. These are included in Figure 1 together with some tangents at the equipotential lines. The reduced gradient curve $E_{x}(x, y)=0$ intersects the equipotential lines in those points where their tangent shows in $x$ direction. Thus, the gradient points in the $y$ direction. It is represented by $\nabla \mathbf{E}=\left(0, E_{y}\right)$. It is a simple but-as we show-effective procedure to follow this curve to determine stationary points. Unlike the usual steepest descent path from a saddle, the reduced gradient search locally has an explicit analytical definition. By the choice of $k$ in eqs. (2) we obtain, in the general case, $N$ different RGF curves passing each stationary point. The existence of these curves follows mathematically if some weak conditions of continuity and differentiability of the PES are fulfilled. We note that these curves are no minimum energy reaction paths. They are defined by the shape of the PES in the given coordinate system, and by the character of the gradient vector between the extrema. But, these curves may follow a reaction valley in favorable cases, at least qualitatively.

The article is organized as follows: In the next section, we illustrate the idea with RGF curves on two-dimensional test surfaces. The outline of the algorithm is then formulated for the general $\mathrm{N}$-dimensional case, which has been incorporated in our version of the GAMESS-UK program. ${ }^{8}$ To demonstrate the method, we examine the $\mathrm{HCN} \rightleftharpoons$ $\mathrm{CNH}$ isomerization. Our results for the PES of this system are presented to show the steplength effectiveness of the RGF. Second, we report the results of the application of the algorithm to the complete six-dimensional PES of formaldehyde, $\mathrm{H}_{2} \mathrm{CO}$, with the STO-3G basis set. The results are compared with those obtained by Bondensgård and Jensen ${ }^{11}$ using another concept. A very different application is the calculation of the saddle point for the isomerization between azidoazomethine and $1 \mathrm{H}$ tetrazole, represented by the SP search using RGF on a 15-dimensional PES. Finally, the relation between RGF and the distinguished coordinate method (coordinate driving procedure) is discussed in more detail.

\section{Discussion of Two-Dimensional Test Surfaces}

With $\mathbf{x}=(x, y)$, the system of eqs. (2) becomes a single equation:

$$
\begin{gathered}
E_{x}(x, y)=0 \text { for } k=2 \text { or } \\
E_{y}(x, y)=0 \text { for } k=1
\end{gathered}
$$

The corresponding RGF curves to find stationary points are calculated by the program Mathematica. ${ }^{12}$ For the illustration of RGF curve properties, we examine:

- The Minyaev-Quapp (MQ) surface ${ }^{13}$ for SP of index 2 of the PES;

- the Müller-Brown (MB) surface ${ }^{14}$;

- the Gonzales-Schlegel (GS) surface ${ }^{15}$ for turning points (TP) of RGF curves;

- the Eckhardt (EC) surface ${ }^{16}$;

- the Neria-Fischer-Karplus (NFK) surface ${ }^{17}$ for bifurcation points (BP) of the RGF and their relation to valley-ridge inflection (VRI) points of the PES.

\section{MINYAEV-QUAPP SURFACE}

The surface ${ }^{13}$ :

$$
\begin{aligned}
E(x, y)=\cos (2 x)+ & 0.57 \cos (2(x-y)) \\
& +\cos (2 y)
\end{aligned}
$$

is given in Figure 2. The points $(\pi, 2 \pi)$ in the upper right corner, and $(0, \pi)$ in the lower left corner are saddle points of index 2. In this example, on the one hand, the RGF curves connect SPs of index 1 with a minimum and, on the other hand, with a saddle of index 2 (a maximum). This behavior may be summarized by the following rule: RGF curves connect StP of even index with StP of odd index, if no BP is transversed.

\section{MÜLLER-BROWN SURFACE-TURNING POINTS}

The PES forms a standard example in theoretical chemistry. ${ }^{14}$ With $A=(-200.0,-100.0$, $-170.0,15.0), a=(-1.0,-1.0,-6.5,0.7), b=(0.0$, $0.0,11.0,0.6), c=(-10.0,-10.0,-6.5,0.7), x^{0}=$ $(1.0,0.0,-0.5,-1.0)$, and $y^{0}=(0.0,0.5,1.5,1.0)$, 


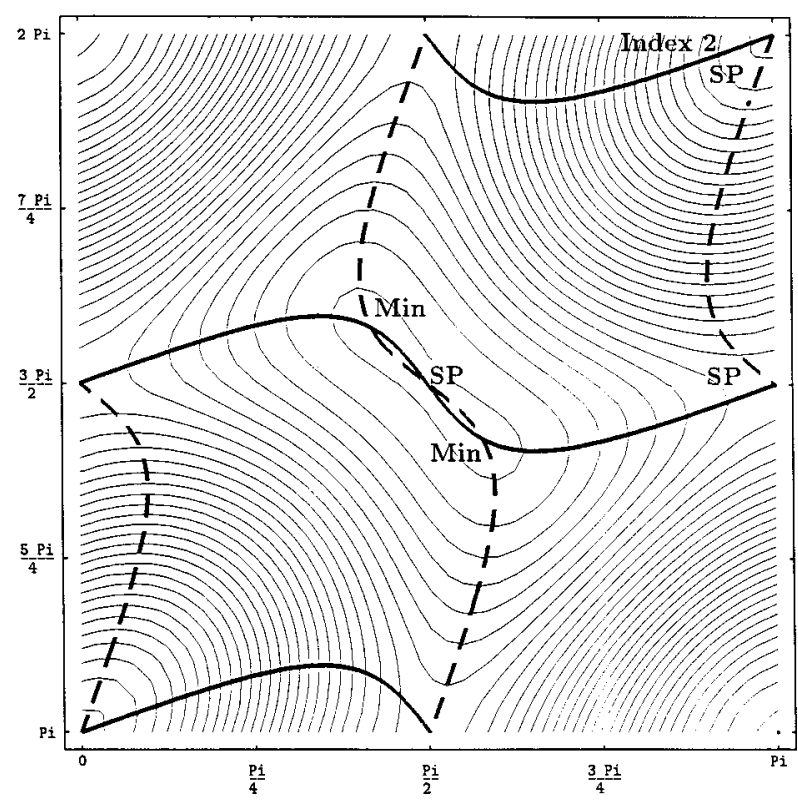

FIGURE 2. Two-dimensional model potential surface $\mathrm{MQ}^{13}$ that also has saddle points of index 2 . They are connected by a reduced gradient curve with saddle points of index 1 (RGF curves $E_{x}=0$ are dashed).

the surface is:

$$
\begin{aligned}
E(x, y)=\sum_{i=1}^{4} A_{i} \exp \left[a_{i}\left(x-x_{i}^{0}\right)^{2}\right. & \\
& \left.\quad+b_{i}\left(x-x_{i}^{0}\right)\left(y-y_{i}^{0}\right)+c_{i}\left(y-y_{i}^{0}\right)^{2}\right]
\end{aligned}
$$

which is displayed in Figure 3. It has a saddle point at $(-0.822,0.624)$, which is not reached by other search strategies if starting at the left minimum $(-0.558,1.442)$ and using only local information. ${ }^{18}$ With the reduced gradient search [eqs. (5)], we find the desired behavior of the two RGF curves: both these curves connect the three minima and two saddles of the PES where they cross. However, the curves do not directly connect the StP by ascending, or by descending the valleys from the starting extrema. They go somewhere along the PES, and show turning points (TP) with respect to the search direction chosen. The TPs reflect the RGF curve back to the valley.

\section{GONZALES-SCHLEGEL SURFACE}

The surface ${ }^{15}$ :

$$
\begin{aligned}
E(x, y)=\operatorname{arccot}[ & \left.-e^{y} \cot (x / 2-\pi / 4)\right] \\
& -2 \exp \left[-0.5(y-\sin x)^{2}\right]
\end{aligned}
$$

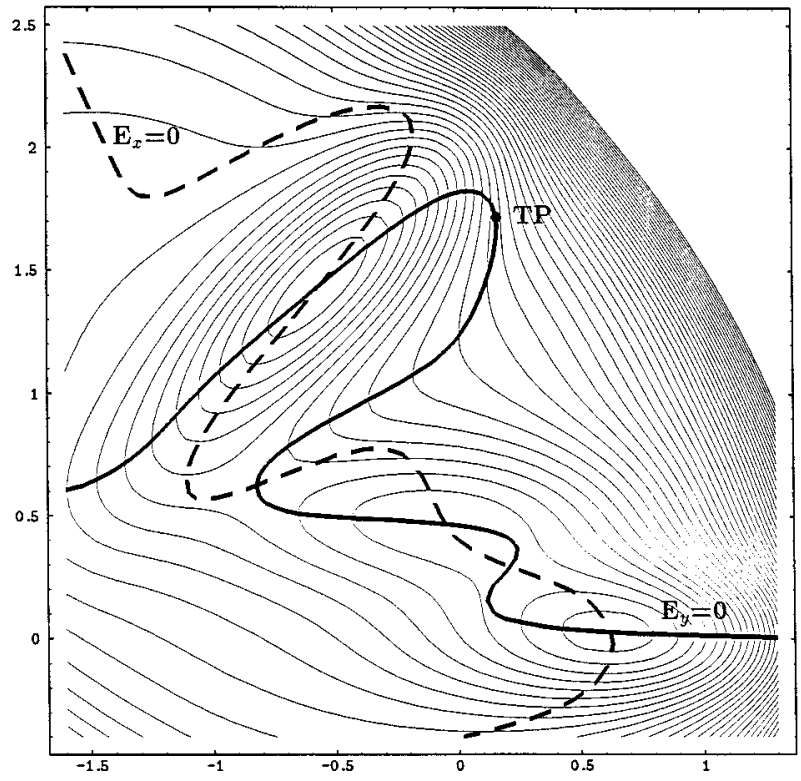

FIGURE 3. The Müller-Brown model potential ${ }^{14}$ with solutions $E_{x}=0$ (dashed) and $E_{y}=0$ (bold). They connect the three minima with the two saddle points. TP marks one of the turning points of the RGF curve $E_{y}=0$.

does not have extrema in the region shown in Figure 4 , but it has a complex valley structure. ${ }^{19 a}$ Starting at point $(4.75,-1)$, the RGF curve $E_{y}=0$, "uphill," nearly perfectly follows the main valley axis arriving at point $(-1.3,-1)$ on the other side

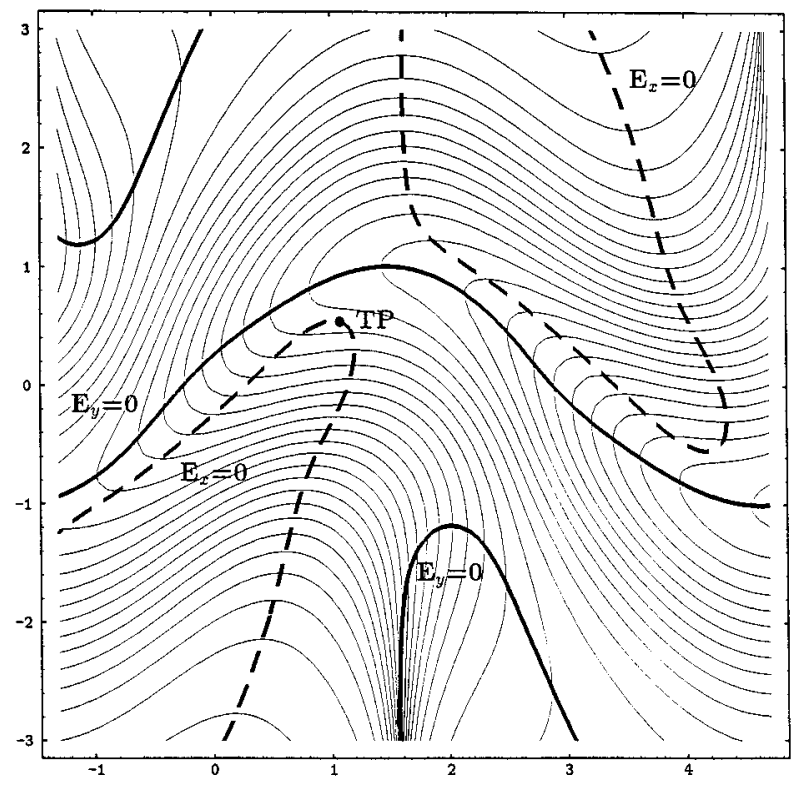

FIGURE 4. Two-dimensional model potential surface GS $^{15}$ showing that RGFs do not necessarily search for the next SP: for $E_{x}=0$ (dashed), the curve leaves the main valley due to a turning point (TP). 
of the panel. We may reverse the search direction and go back "downhill" from $(-1.3,-1)$ along the same pathway. However, if we start at a point nearby, at $(-1.3,-1.3)$, and search for the other RGF curve $E_{x}=0$ (dashed), we trace along a curve near the valley axis, going downhill quite similarly to the former pathway as far as the point $\approx(1,0.5)$. The curve then leaves the main valley due to a TP and subsequently follows a ridge up to another SP.

\section{ECKHARD'T SURFACE-VALLEY-RIDGE INFLECTION POINTS}

The test surface $^{16}$ is given in Figure 5. The formula is:

$$
\begin{aligned}
E(x, y)= & \exp \left(-x^{2}-(y+1)^{2}\right) \\
& +\exp \left(-x^{2}-(y-1)^{2}\right) \\
& +4 \exp \left(-3\left(x^{2}+y^{2}\right) / 2\right)+y^{2} / 2
\end{aligned}
$$

Here, in contrast to the Müller-Brown example, the solutions of eqs. (5) follow the valleys or ridges defined by eq. (9). This example is interesting because it shows another possibility of the RGF

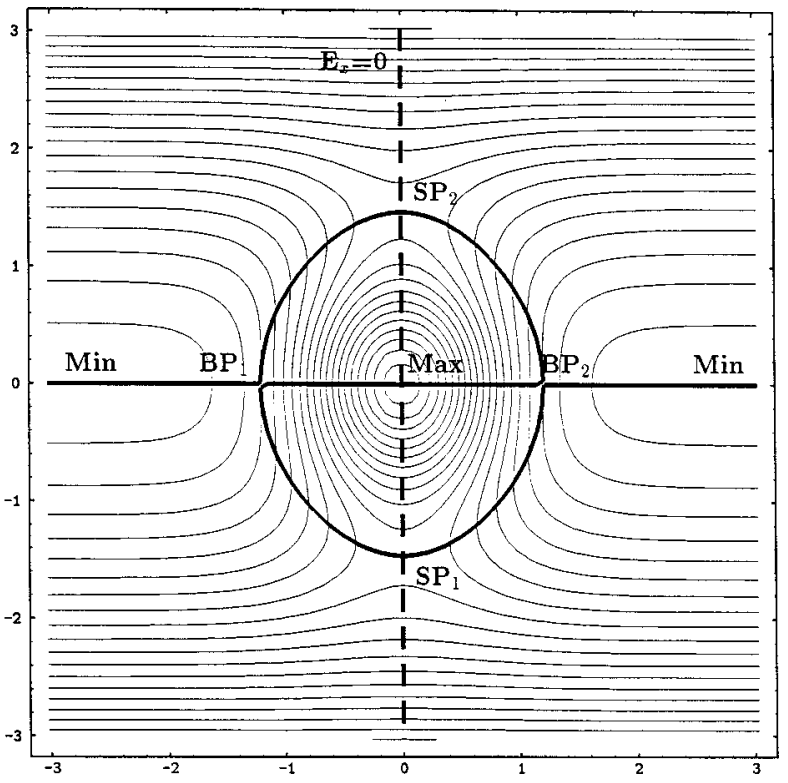

FIGURE 5. Two-dimensional model potential surface $\mathrm{EC}^{16}$ with minimum (Min), maximum (Max), saddles (SP) of the surface, and two bifurcation points (BP) on the bold RGF curve $E_{y}=0$. The BPs are valley-ridge inflection points of the surface. The roundabout path from $\mathrm{SP}_{1}$ to $\mathrm{BP}_{1}$ to $\mathrm{SP}_{2}$ to $\mathrm{BP}_{2}$, and back to $\mathrm{SP}_{1}$ connects only the SPs, but does not cross a minimum or a maximum. curve defined by the nonlinear equation [eq. (2)]: It can cross a BP between the extrema. If there are such points, a particular property of RGF becomes evident. The RGF curve for $E_{y}=0$ (bold) along the symmetry axis from the minimum to the maximum does not cross an SP of index 1, but directly leads to a saddle of index 2. (If the PES shows symmetry, and if a search direction of RGF reflects this symmetry, then the RGF search holds this symmetry.) A second curve for $E_{y}=0$ starting in $\mathrm{SP}_{1}$ goes to $\mathrm{SP}_{2}$ without touching the minimum or the maximum. The BP only emerges if two RGF curves of the same search direction meet, here for $E_{y}=0$. Following the symmetry axis from the minimum to the maximum, a qualitative change of the curvature of equipotential lines orthogonal to this axis occurs at the BP. Convexity changes to concavity. We derive the hypothesis that the point of change describes the so-called valley-ridge inflection (VRI) point of the surface. In this manner, at least for the symmetric case, RGF is able to locate VRI points. For a discussion of VRI and its chemical importance see refs 18-20.

\section{NERIA-FISCHER-KARPLUS SURFACE}

In Figure 6 the function ${ }^{17}$ :

$$
\begin{aligned}
E(x, y)= & 0.06\left(x^{2}+y^{2}\right)^{2}+x y \\
& -9 \exp \left(-(x-3)^{2}-y^{2}\right) \\
& -9 \exp \left(-(x+3)^{2}-y^{2}\right)
\end{aligned}
$$

is displayed. It has no symmetry plane, but rather $\mathrm{C}_{2}$ symmetry. In this particular case, we use another ansatz of the reduced gradient idea: We do not set one component part of the gradient to zero; that is, we do not assign the search direction to one of the coordinates, $k=1$ or 2 . In contrast, a curve for a general search direction $\mathbf{q}$; that is, with $\nabla E(\mathbf{x}) \| \mathbf{q}$ is traced. If $\mathbf{q}=\left(q_{x}, q_{y}\right)$, the orthogonal vector to $\mathbf{q}$ will be $\mathbf{q}^{\perp}=\left(q_{y},-q_{x}\right)$, and the gradient $\nabla E(\mathbf{x})$ will also be orthogonal to this vector. We use the slightly more complicated scalar product equation:

$$
\nabla E(\mathbf{x}) \mathbf{q}^{\perp}=E_{x} q_{y}-E_{y} q_{x}=0
$$

as a modified RGF instead of eqs. (2). Modified RGF solutions of the NFK surface are included in Figure 6. The search directions are the vectors $\pm\left(q_{x}, q_{y}\right)= \pm(0.47924,0.52076)$ starting at the minima. Only in this special case do we obtain a solution going through the BPs at $(1.55,1.95)$ and 


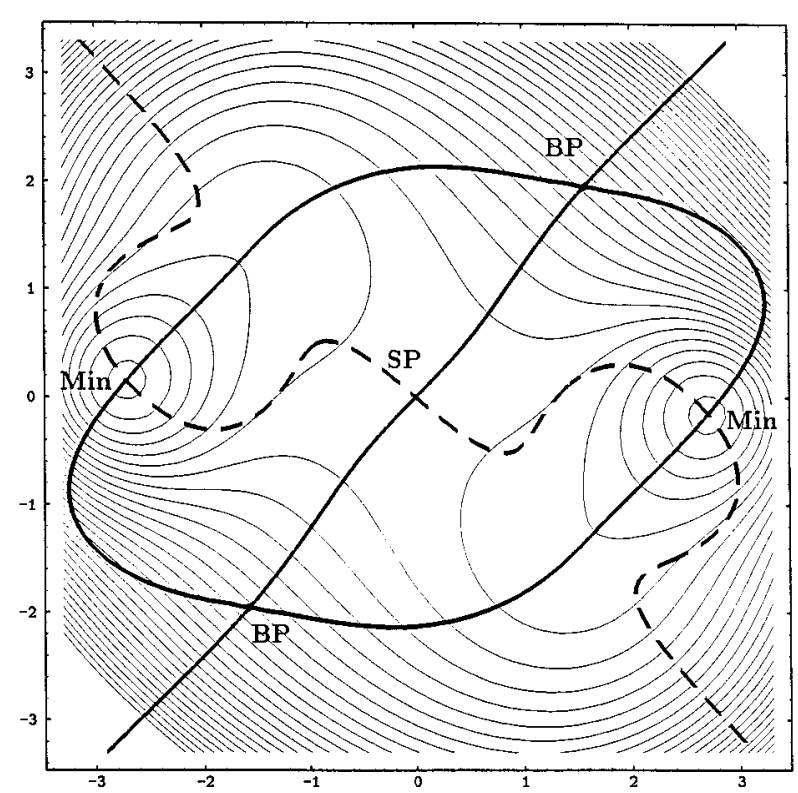

FIGURE 6. Two-dimensional model potential surface $\mathrm{NFK}^{17}$ with bifurcation points (BP) on a modified reduced gradient curve. The roundabout path from the two minima over the two bifurcation points does not touch a saddle point. A modification of the method, using combined search directions, is used. The BP of this RGF is a valley-ridge inflection point of the surface.

$(-1.55,-1.95)$ and connecting two minima without intersecting the SP. The other search direction (dashed), $q_{x}=0.48$ and $q_{y}=-0.52$, leads to the SP $(0,0)$ if we start in the left minimum. Note that the intersection of the two RGF curves at the BP is not orthogonal. This bifurcation happens in a nonsymmetrical surface region. (Such a skew bifurcation is also possible for other curves; e.g., compare with gradient extremals [GE]. ${ }^{21}$ ) In the NFK surface, again, the bifurcation point, indicated by the RGF curves, is a valley-ridge inflection point (VRI) of the surface. The ridge leading from the SP to the $\mathrm{BP}$ changes at the BP into a valley going further uphill. Taking the opposite view, the downhill valley path from the upper right corner of the panel is tripled at the BP into two valleys leading to the two minima, and the ridge in between. This threefold branching pattern of curves is a so-called pitchfork bifurcation. Because the surface is unsymmetrical, the pitchfork is unsymmetrical as well.

This surface (Fig. 6) shows additionally the general advantages of the RGF method. We may choose any search direction $\tilde{\mathbf{q}}$ leaving the minima inside the range of the curvilinear rhomboid connecting the two minima and the two bifurcation points. Such a search direction, $\tilde{\mathbf{q}}$, will then successfully find the SP at $(0,0)$. This is shown for the dashed RGF pathway with $q_{x}=0.48$ and $q_{y}=$ -0.52 starting in the left minimum. The paths obtained in this way by a different search direction $\tilde{\mathbf{q}}$ inside the rhomboid do not pass the BP, but they may go through a TP. With respect to the general case to find an initial guess to reach an SP or a VRI, it cannot be expected to find exactly that direction $\mathbf{q}$ which generates the RGF curve with the branching point along the ridge of the PES. It thus remains a difficult task to find an unsymmetric $\mathrm{BP}$ (i.e., VRI point) even by the use of this approach. (The theory of gradient extremals ${ }^{18,21,22}$ allows that ridge to be determined. However, in this example, the GEs do not have a BP at all.)

\section{Algorithm}

\section{PREDICTOR STEP}

We assume a curve of points, $\mathbf{x}(t)$, fulfilling the $N-1$ equations:

$$
\frac{\partial E(\mathbf{x}(t))}{\partial x^{i}}=0, \quad i=1, \ldots, k \ldots, N
$$

however, $\frac{\partial E(\mathbf{x}(t))}{\partial x^{k}} \neq 0$ outside stationary points

The parameter $t$ varies in a certain interval. The starting point is any stationary point. To predict the corresponding next point, we calculate the tangent to the curve. ${ }^{23}$ It is given by:

$$
\begin{aligned}
& \frac{d}{d t} \frac{\partial E(\mathbf{x}(t))}{\partial x^{i}}= 0=\sum_{l=1}^{N} \frac{\partial^{2} E(\mathbf{x})}{\partial x^{i} \partial x^{l}} \frac{d x^{l}(t)}{d t}, \\
& i=1, \ldots, k \ldots, N, \text { or } \\
& \hat{\mathbf{H}} \mathbf{x}^{\prime}=\mathbf{0}
\end{aligned}
$$

It is a homogeneous system of $N-1$ linear equations for the direction cosine of the $N$ components, $d x^{l} / d t$, of the tangent, $\mathbf{x}^{\prime}$. The coefficients are entries of the Hessian matrix, $\hat{\mathbf{H}}=\partial^{2} E(\mathbf{x}) / \partial x^{i} \partial x^{l}$, where the $i=k$ th row is omitted. We may use an update procedure for the Hessian ${ }^{1}$ (see below). If internal coordinates are used, in particular curvilinear coordinates, the corresponding formulas of the metric tensor have to be included. ${ }^{1,2,5,24}$ The algorithm uses $Q R$ decomposition of the matrix of system (13) to obtain the solution. ${ }^{23}$ ( $Q$ is an orthogonal matrix, $R$ is an upper triangular matrix.) 
The predictor step is:

$$
\mathbf{x}_{\mathrm{m}+\mathbf{1}}=\mathbf{x}_{\mathbf{m}}+\frac{S t L}{\left\|\mathbf{x}_{\mathbf{m}}^{\prime}\right\|} \mathbf{x}_{\mathbf{m}}^{\prime}
$$

where $m$ indicates the number of calculated points, and the steplength, $S t L$, is used as a parameter in the algorithm. For example, we take $S t L=0.1$ units of the corresponding coordinate $(\AA, \mathrm{rad})$ in the case of the four-atom $\mathrm{H}_{2} \mathrm{CO}$, and we use from 0.2 up to 0.6 units for tetrazole with seven atoms. The test case $\mathrm{HCN}$ is given with $0.1-0.9 \mathrm{rad} S t L$.

Curves having the same property as the solutions of eq. (13), thus showing in every point a fixed gradient direction of the PES, are also obtained by Branin's differential equation. ${ }^{25}$ The results reached by the RGF algorithm depend on the selected direction, the so-called distinguished coordinate, as well as on the set of internal coordinates defined by the $\mathbf{Z}$ matrix. Care must be exercised in this choice, as well as in the starting direction of the search. It is quite normal for RGF that turning points may occur (if the type D surface of Williams and Maggiora ${ }^{10}$ is met). Using the tangent search [eq. (13)], the algorithm goes through a TP without problems, because we do not minimize orthogonal to the distinguished coordinate. In contrast to the older method, we solve the well-posed system of eqs. (13). If at any point arrived at by RGF, eq. (12) is fulfilled to a given tolerance, the next predictor step is executed, otherwise the algorithm skips to the corrector, as shown in Figure 7. If the SCF part of the calculation does not converge, and if the stability and continuity of the wave functions are lost, the search for the next point is stopped.

\section{CORRECTOR STEP}

A Newton-Raphson-like method is used to solve the reduced system of eqs. (12). The steplength of this method is given intrinsically, and it is used until convergence. However, in addition, we impose an upper limit on the steplength, because, if a bifurcation point is touched, the pure Newton corrector produces steps that are too large. The tolerance of the corrector is $0.1 \times$ (predictor StL).

\section{STOPPING CRITERION}

At every point along the pathway of the search we determine the steplength of a hypothetical Newton step to the "next" stationary point. If this
Scheme of the Reduced Gradient Following algorithm

\begin{tabular}{|l|l|}
\hline \multicolumn{2}{|c|}{ Initialisation, preparing and execution of the first step } \\
\hline Transformation from internal to Cartesian coordinates \\
\hline Calculation of energy, gradient and Hessian (exact or updated) \\
\hline Mass weighting \\
\hline Calculation of the metric tensor, $\mathrm{g}$, and the matrix of coordinate transformation, $\mathrm{B}$ \\
\hline Transformation of gradient and Hessian into internal coordinates \\
\hline Vibrational analysis in internal coordinates \\
\hline Calculation of the Newton-Raphson step \\
\hline Reducing of gradient and Hessian by the selected coordinate \\
\hline Calculation of tangent by $\mathrm{QR}$-decomposition \\
\hline Setting of an augmented matrix $\mathrm{K}$ by addition of the tangent to the reduced Hessian \\
\hline Predictorstep \\
\hline Calculation of arclength of the curve \\
\hline Test of bifurcation and turning points \\
\hline Storing of the actual values \\
\hline Execution of the step \\
\hline Repeat until stopping criterion is satisfied \\
\hline
\end{tabular}

FIGURE 7. Schematic flowchart of the reduced gradient following (RGF) algorithm.

value falls below a specified tolerance, say $(0.5+$ $\epsilon) \times($ predictor $S t L)$, the algorithm still carries out this step in the endgame, and then stops.

There are options to avoid unnecessary calculations. We next describe two different strategies.

\section{DYNAMICAL STEPLENGTH}

Usually, the corrector is used very sparsely by the algorithm. If the predictor agrees "exactly" with the curve direction (with a tolerance of $5^{\circ}$ ), we increase $S t L$ of the predictor by the factor $\sqrt{2} .^{26}$ The $S t L$ is decreased by $1 / \sqrt{2}$ if a corrector step is called.

\section{UPDATE OF HESSIAN MATRIX}

We found that the algorithm is very stable against update procedures of the Hessian matrix. The reduced gradient criterion of eqs. (12) needs only the gradient, and thus it can be used exactly. Of course, if we connect StPs of a different index by RGF, an update must be taken allowing such changes of the index of the Hessian. This is guaranteed by the update of Davidon ${ }^{27}$ and Fletcher and Powell, ${ }^{28}$ the so-called DFP update. ${ }^{1}$ In the examples, we need one calculation of the exact Hessian at the starting point of the path, and a second one at the end in order to proof the index of the StP reached. If a BP is met along the path, we recommend a further exact calculation of the Hessian because the corrector step may diverge. 


\section{Examples from Chemistry}

\section{HCN $\rightleftharpoons$ CNH ISOMERIZATION}

We present this unimolecular reaction ${ }^{29}$ as a first test using a real chemical system. In this case, the distinguished reaction coordinate is the bending coordinate. It roughly describes the minimum energy path along a deep valley of the PES. The arclength of that valley is approximately $3 \mathrm{rad}$ between the HCN minimum and SP, and $4 \mathrm{rad}$ between HNC and SP. A fixed StL in eq. (14) was examined between 0.1 and $0.9 \mathrm{rad}$. The number of predictor steps $(\mathrm{P})$ is given in Table I using the RHF/6-311G** basis set within the GAMESS-UK package. ${ }^{8}$ In all cases, the corrector was not required, because of the simplicity of the pathway. The points of the RGF curve lead from the HCN minimum over the well-known SP to the HNC minimum or vice versa. The number of steps needed by RGF is obviously the minimal number to measure the arclength of the pathway. Using the DFP update under the same (fixed) StLs, we obtain similar results where a moderate number of corrector steps is additionally called. If no turning

\section{TABLE I.}

\section{RGF Test Along the Bending Coordinate for $\mathrm{HCN} \rightleftharpoons \mathrm{CNH}$ Isomerization. ${ }^{\mathrm{a}}$}

\begin{tabular}{|c|c|c|c|c|}
\hline \multirow{2}{*}{$\begin{array}{l}\text { Start in HCN: } \\
\text { Hessian } \\
\text { steplength }\end{array}$} & \multicolumn{2}{|c|}{ Pathway to SP } & \multicolumn{2}{|c|}{$\begin{array}{c}\text { Pathway } \\
\text { from HNC to SP }\end{array}$} \\
\hline & $\begin{array}{c}\text { Exact } \\
\mathrm{P}^{\mathrm{b}}\end{array}$ & $\begin{array}{l}\text { Update } \\
\mathrm{P}^{\mathrm{b}} / \mathrm{C}\end{array}$ & $\begin{array}{c}\text { Exact } \\
\mathrm{P}^{\mathrm{b}}\end{array}$ & $\begin{array}{l}\text { Update } \\
\mathrm{Pb}^{\mathrm{b}} / \mathrm{C}\end{array}$ \\
\hline $0.1 \mathrm{rad}$ & 33 & $32 / 1$ & 41 & $36 / 3$ \\
\hline $0.2 \mathrm{rad}$ & 16 & $16 / 2$ & 21 & $18 / 3$ \\
\hline $0.3 \mathrm{rad}$ & 11 & $11 / 2$ & 14 & $12 / 3$ \\
\hline $0.4 \mathrm{rad}$ & 8 & $8 / 1$ & 11 & $11 / 3$ \\
\hline $0.5 \mathrm{rad}$ & 6 & $6 / 1$ & 8 & $7 / 8$ \\
\hline $0.6 \mathrm{rad}$ & 5 & $5 /-$ & 6 & $6 / 2$ \\
\hline $0.7 \mathrm{rad}$ & 5 & $5 / 1$ & 5 & $5 / 2$ \\
\hline $0.8 \mathrm{rad}$ & 4 & $5 / 3$ & 5 & $5 / 2$ \\
\hline $0.9 \mathrm{rad}$ & 4 & $5 / 2$ & 5 & $5 / 2$ \\
\hline
\end{tabular}

\begin{tabular}{lcccc}
\hline$S t P^{c}$ & $E$ (a.u.) & $r_{\mathrm{HC}}(\AA)$ & $r_{\mathrm{CN}}(\AA)$ & $\angle \mathrm{HCN}$ (deg.) \\
\hline $\mathrm{HCN}$ & -92.83259 & 1.14425 & 1.05198 & 180.000 \\
$\mathrm{SP}$ & -92.73475 & 1.18466 & 1.16957 & 74.042 \\
$\mathrm{HNC}$ & -92.82146 & 1.16586 & 2.14615 & 0.000
\end{tabular}

${ }^{a} \mathrm{P}$ is the number of predictor points, $\mathrm{C}$ those of the corrector.

${ }^{\mathrm{b}}$ Including the start point with exact Hessian.

${ }^{\mathrm{c}} \mathrm{RHF} / 6-311 \mathrm{G} * *$. or bifurcation point is met, as in this case, the computational cost of the method decreases with increasing steplength of the predictor.

The calculation of the pathway between SP and HNC with the dynamical steplength option of the predictor, beginning with $0.1 \AA$ St $L$, needs 10 predictor points and 1 corrector. By using both of the options, update + dynamical step length, we can calculate the pathway by 17 predictor steps and 8 correctors.

\section{FULL SIX-DIMENSIONAL PES OF FORMALDEHYDE}

We have mapped out the RGF curves using the RHF/STO-3G potential energy surface of $\mathrm{H}_{2} \mathrm{CO}$. The GAMESS-UK program was used. ${ }^{8}$ There are five previously specified structures corresponding to minima: formaldehyde; cis- and trans-hydroxycarbene; $\mathrm{H}_{2}+\mathrm{CO}$ as dissociation products ${ }^{20 \mathrm{~b}, 30-32}$; and the $\mathrm{COH}_{2}$ isomer. ${ }^{11}$ They are in agreement with chemical intuition. However, our calculations on $\mathrm{H}_{2} \mathrm{CO}$ aim at a first analysis of the power of the search algorithm. The RHF/STO-3G level of theory was chosen for comparison with ref. 11 and cannot chemically describe certain dissociation processes.

We start at the global minimum, $\mathrm{M}_{1}$, of $\mathrm{H}_{2} \mathrm{CO}$ as well as at the other minima with a systematic search along all mass-weighted curvilinear internal coordinates of the six-dimensional PES with a positive or negative initial steplength. For computational control, the directions which do not belong to the totally symmetric representation of the point group are also followed in both initial directions. Several different schemes of $\mathbf{Z}$ matrices are used, which are listed in Table II; see also Figure 8 for the definition of the coordinates. We define a total of 12 RGF curves that are followed from each stationary point. If a new stationary point is de-

TABLE II.

Different $Z$ Matrices Used in $\mathrm{H}_{2} \mathrm{CO}$ Calculation. ${ }^{\mathrm{a}}$

\begin{tabular}{ccccccc}
\hline No. & $r_{1}$ & $r_{2}$ & $r_{3}$ & $\alpha_{1}$ & $\alpha_{2}$ & $\theta$ \\
\hline 1 & $r_{\mathrm{CO}}$ & $r_{\mathrm{CH}_{1}}$ & $r_{\mathrm{CH}_{2}}$ & $\angle \mathrm{H}_{1} \mathrm{CO}$ & $\angle \mathrm{H}_{2} \mathrm{CO}$ & $\angle \mathrm{H}_{2} \mathrm{COH}_{1}$ \\
2 & $r_{\mathrm{CO}}$ & $r_{\mathrm{OH}_{1}}$ & $r_{\mathrm{OH}_{2}}$ & $\angle \mathrm{H}_{1} \mathrm{OC}$ & $\angle \mathrm{H}_{2} \mathrm{OC}$ & $\angle \mathrm{H}_{2} \mathrm{OCH}_{1}$ \\
3 & $r_{\mathrm{CO}}$ & $r_{\mathrm{CH}_{1}}$ & $r_{\mathrm{OH}_{2}}$ & $\angle \mathrm{H}_{1} \mathrm{CO}$ & $\angle \mathrm{H}_{2} \mathrm{OC}$ & $\angle \mathrm{H}_{2} \mathrm{OCH}_{1}$ \\
4 & $r_{\mathrm{H}_{1} \mathrm{O}}$ & $r_{\mathrm{H}_{1} \mathrm{C}}$ & $r_{\mathrm{H}_{1} \mathrm{H}_{2}}$ & $\angle \mathrm{OH}_{1} \mathrm{C}$ & $\angle \mathrm{H}_{2} \mathrm{H}_{1} \mathrm{C}$ & $\angle \mathrm{H}_{2} \mathrm{H}_{1} \mathrm{CO}$ \\
5 & $r_{\mathrm{H}_{1} \mathrm{C}}$ & $r_{\mathrm{H}_{1} \mathrm{H}_{2}}$ & $r_{\mathrm{H}_{1} \mathrm{O}}$ & $\angle \mathrm{H}_{2} \mathrm{H}_{1} \mathrm{C}$ & $\angle \mathrm{OH}_{1} \mathrm{H}_{2}$ & $\angle \mathrm{OH}_{1} \mathrm{H}_{2} \mathrm{C}$ \\
6 & $r_{\mathrm{CH}_{1}}$ & $r_{\mathrm{CH}_{2}}$ & $r_{\mathrm{CO}}$ & $\angle \mathrm{H}_{2} \mathrm{CH}_{1}$ & $\angle \mathrm{OCH}_{2}$ & $\angle \mathrm{OCH}_{2} \mathrm{H}_{1}$ \\
7 & $r_{\mathrm{OH}_{1}}$ & $r_{\mathrm{OH}_{2}}$ & $r_{\mathrm{OC}}$ & $\angle \mathrm{H}_{2} \mathrm{OH}_{1}$ & $\angle \mathrm{COH}_{2}$ & $\angle \mathrm{COH}_{2} \mathrm{H}_{1}$ \\
\hline \multicolumn{7}{c}{ Compare with Figure 8. } \\
\multicolumn{7}{c}{ Compare wing }
\end{tabular}




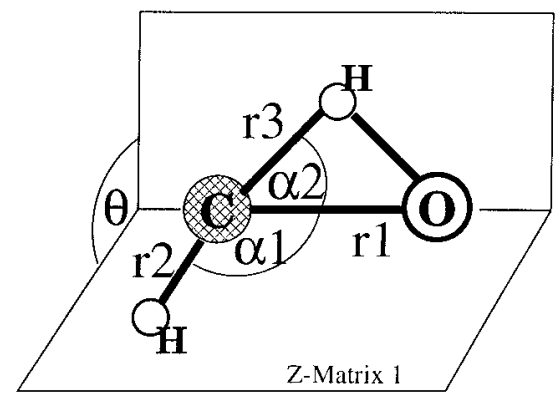

FIGURE 8. Illustration of a $\mathbf{Z}$ matrix defining the internal coordinates in the calculations of $\mathrm{H}_{2} \mathrm{CO}$.

tected, it is recalculated by a Newton-Raphson fit with a smaller tolerance, and it is used as a new starting point. This systematic search locates a total of 7 minima, 13 SPs of index 1, 20 SPs of index 2, and 9 SPs of index 3 . The results are given in Table III. The stationary points are differentiated by their index, and are numbered by a subscript $i$ in order of the energy: we use $\mathrm{M}_{i}$ for minima, $\mathrm{F}_{i}$ for first index saddle points, $S_{i}$ for the second index, and $T_{i}$ for the third index saddles. Higher index saddles are not detected. We located all of the stationary points found by Bondensgård and
Jensen. ${ }^{11}$ Those detected in addition to these are marked by an asterisk. About 14 of the stationary points are loosely bound complexes of the van der Waals type. About seven of the new structures are more strongly bonded. New stationary points are recalculated at the MP2/6-31+ $\mathrm{G}^{* *}$ level to check for chemical relevance. Some of them are put to the proof, as will be discussed in what follows. Figure 9 gives an overview of some stationary points and the way to reach them by RGF. The coordinate used for the successful RGF search is also indicated.

The information contained in the rich pattern of StP on the PES of formaldehyde can now be surveyed. The transition structures corresponding to formaldehyde dissociation into $\mathrm{H}_{2}+\mathrm{CO}\left(\mathrm{M}_{2}\right)$ and the trans-hydroxycarbene case $\left(\mathrm{M}_{3}\right)$ have been well studied previously. ${ }^{20 \mathrm{~b}, 30-32}$ The nonsymmetric saddle $\mathrm{F}_{4}$, to dissociation into $\mathrm{H}_{2}+\mathrm{CO}$, is reached starting in $\mathrm{M}_{1}$ with direction $\alpha_{1}^{+}$, and the dissociation from $\mathrm{F}_{4}$ to $\mathrm{M}_{2}$ is obtained with an increasing $r_{1}$. The formaldehyde $\left(\mathrm{M}_{1}\right)$ isomerization to transhydroxycarbene $\left(\mathrm{M}_{3}\right)$ takes place via the nonsymmetric saddle $F_{2}$. The usual minimum energy path of this isomerization takes place via unequal

TABLE III.

Stationary Points (StP) of the PES of Formaldehyde Using the RHF / STO-3G Level (New Structures Marked with Asterisk).

\begin{tabular}{|c|c|c|c|c|c|c|c|c|c|}
\hline$S t P^{a}$ & $\mathbf{Z}$ mat. $^{\mathrm{b}}$ & $\begin{array}{c}\text { Energy } \\
\text { (a.u.) }\end{array}$ & Symm. & $\begin{array}{l}r_{1} \\
(\AA)\end{array}$ & $\begin{array}{l}r_{2} \\
(\AA)\end{array}$ & $\begin{array}{l}r_{3} \\
(\AA)\end{array}$ & $\begin{array}{c}\alpha_{1} \\
\text { (deg.) }\end{array}$ & $\begin{array}{c}\alpha_{2} \\
\text { (deg.) }\end{array}$ & $\begin{array}{c}\theta \\
\text { (deg.) }\end{array}$ \\
\hline$M_{1}$ & 1 & -112.3544 & $\mathrm{C}_{2 v}$ & 1.21672 & 1.10139 & 1.10139 & 122.738 & 122.738 & 180.0 \\
\hline $\mathrm{M}_{2}^{\mathrm{C}}$ & - & -112.3429 & - & 1.14547 & 0.71215 & - & - & - & - \\
\hline $\mathrm{M}_{3}$ & 3 & -112.2784 & $\mathrm{C}_{s}$ & 1.33127 & 1.12941 & 0.99058 & 100.825 & 108.136 & 180.0 \\
\hline$M_{4}$ & 3 & -112.2691 & $\mathrm{C}_{s}$ & 1.32641 & 1.13139 & 0.99388 & 106.101 & 114.211 & 0.0 \\
\hline$M_{5}$ & 2 & -112.0780 & $\mathrm{C}_{s}$ & 1.71987 & 0.98660 & 0.98660 & 119.640 & 119.640 & 131.454 \\
\hline$M_{6}^{*}$ & 5 & -112.0610 & $\mathrm{C}_{s}$ & 2.16510 & 1.51985 & 0.99075 & 140.168 & 39.797 & 180.0 \\
\hline $\mathrm{M}_{7}^{*}$ & 3 & -112.0338 & $\mathrm{C}_{2 v}$ & 2.82299 & 1.12307 & 2.14433 & 57.942 & 21.009 & 180.0 \\
\hline$F_{1}$ & 3 & -112.2321 & $\mathrm{C}_{1}$ & 1.40187 & 1.14078 & 0.99319 & 103.129 & 103.502 & 89.197 \\
\hline $\mathrm{F}_{2}$ & 3 & -112.1648 & $\mathrm{C}_{1}$ & 1.33300 & 1.12257 & 1.30256 & 107.851 & 55.950 & 111.962 \\
\hline $\mathrm{F}_{3}$ & 6 & -112.1470 & $\mathrm{C}_{s}$ & 1.05340 & 1.92162 & 1.30595 & 152.412 & 29.248 & 180.0 \\
\hline $\mathrm{F}_{4}$ & 1 & -112.1291 & $\mathrm{C}_{s}$ & 1.20045 & 1.11706 & 1.48908 & 155.073 & 106.339 & 0.0 \\
\hline $\mathrm{F}_{5}$ & 1 & -112.1021 & $\mathrm{C}_{s}$ & 1.27767 & 1.48378 & 1.45857 & 90.902 & 50.381 & 0.0 \\
\hline $\mathrm{F}_{6}$ & 2 & -112.0775 & $\mathrm{C}_{2 v}$ & 1.67836 & 0.98585 & 0.98583 & 126.862 & 126.862 & 180.034 \\
\hline $\mathrm{F}_{7}^{*}$ & 5 & -112.0581 & $\mathrm{C}_{2 v}$ & 2.77934 & 1.50582 & 0.98850 & 74.283 & 40.388 & 180.0 \\
\hline $\mathrm{F}_{8}^{*}$ & 3 & -112.0575 & $\mathrm{C}_{1}$ & 2.93626 & 2.48670 & 0.98896 & 18.756 & 116.857 & 82.978 \\
\hline$F_{9}$ & 3 & -112.0466 & $\mathrm{C}_{s}$ & 1.73316 & 1.39764 & 0.99045 & 38.280 & 102.573 & 100.674 \\
\hline $\mathrm{F}_{10}^{*}$ & 2 & -111.9727 & $\mathrm{C}_{1}$ & 1.35832 & 1.04344 & 1.33792 & 111.854 & 120.450 & 70.685 \\
\hline$F_{11}$ & 2 & -111.9323 & $\mathrm{C}_{s}$ & 1.34529 & 1.20744 & 1.43876 & 65.309 & 114.775 & 0.0 \\
\hline $\mathrm{F}_{12}^{*}$ & 3 & -111.9081 & $\mathrm{C}_{2 v}$ & 1.20371 & 1.80221 & 1.51869 & 56.567 & 82.024 & 180.0 \\
\hline$F_{13}^{*}$ & 3 & -111.8698 & $\mathrm{C}_{s}$ & 3.32963 & 2.40530 & 1.55819 & 26.559 & 70.008 & 0.0 \\
\hline \multicolumn{10}{|c|}{ (Continued) } \\
\hline
\end{tabular}


TABLE III.

\section{(Continued)}

\begin{tabular}{|c|c|c|c|c|c|c|c|c|c|}
\hline$S t P^{a}$ & $\mathbf{Z}$ mat. $^{\text {b }}$ & $\begin{array}{c}\text { Energy } \\
\text { (a.u.) }\end{array}$ & Symm. & $\begin{array}{l}r_{1} \\
(\AA)\end{array}$ & $\begin{array}{l}r_{2} \\
(\AA)\end{array}$ & $\begin{array}{l}r_{3} \\
(\AA)\end{array}$ & $\begin{array}{c}\alpha_{1} \\
\text { (deg.) }\end{array}$ & $\begin{array}{c}\alpha_{2} \\
\text { (deg.) }\end{array}$ & $\begin{array}{c}\theta \\
\text { (deg.) }\end{array}$ \\
\hline$S_{1}$ & 7 & -112.1990 & $\mathrm{C}_{s}$ & 0.95617 & 1.92005 & 1.29571 & 145.951 & 35.616 & 180.0 \\
\hline $\mathrm{S}_{2}$ & 3 & -112.1497 & $\mathrm{C}_{s}$ & 1.29971 & 1.11537 & 1.20053 & 116.882 & 57.239 & 179.995 \\
\hline $\mathrm{S}_{3}$ & 6 & -112.0722 & $C_{1}$ & 1.16106 & 1.25123 & 1.32900 & 65.156 & 78.695 & 242.254 \\
\hline $\mathrm{S}_{4}$ & 1 & -112.0702 & $\mathrm{C}_{1}$ & 1.31305 & 1.24781 & 1.19933 & 93.668 & 89.624 & 60.741 \\
\hline $\mathrm{S}_{5}^{*}$ & 3 & -112.0574 & $\mathrm{C}_{s}$ & 2.95089 & 2.59530 & 0.98885 & 19.187 & 160.056 & 0.0 \\
\hline $\mathrm{S}_{6}^{*}$ & 3 & -112.0571 & $\mathrm{C}_{s}$ & 3.07838 & 2.87698 & 0.98885 & 18.722 & 69.046 & 250.095 \\
\hline $\mathrm{S}_{7}$ & 1 & -112.0341 & $\mathrm{C}_{s}$ & 1.40844 & 1.07178 & 1.24713 & 165.634 & 50.691 & 0.0 \\
\hline $\mathrm{S}_{8}$ & 3 & -112.0302 & $\mathrm{C}_{s}$ & 2.06846 & 1.11678 & 1.33352 & 72.820 & 31.950 & 180.0 \\
\hline $\mathrm{S}_{9}^{\circ}$ & 3 & -112.0219 & $\mathrm{C}_{s}^{s}$ & 1.53672 & 1.52786 & 0.99284 & 39.264 & 143.858 & 180.0 \\
\hline$S_{10}$ & 3 & -112.0204 & $\mathrm{C}_{s}$ & 1.80830 & 1.30049 & 0.99856 & 38.674 & 135.520 & 0.0 \\
\hline$S_{11}$ & 3 & -112.0155 & $\mathrm{C}_{s}$ & 1.91911 & 1.26391 & 0.98630 & 34.627 & 79.529 & 180.0 \\
\hline $\mathrm{S}_{12}^{*}$ & 3 & -111.9710 & $\mathrm{C}_{s}$ & 1.38911 & 2.17343 & 1.16417 & 28.679 & 116.377 & 66.663 \\
\hline $\mathrm{S}_{13}$ & 3 & -111.9676 & $\mathrm{C}_{2 v}$ & 1.86102 & 1.27492 & 1.18833 & 39.213 & 42.709 & 180.0 \\
\hline$S_{14}^{*}$ & 3 & -111.9569 & $\mathrm{C}_{\infty V}$ & 2.79129 & 1.83811 & 0.93538 & 0.0 & 180.0 & 180.0 \\
\hline$S_{15}$ & 2 & -111.9549 & $\mathrm{C}_{s}$ & 1.25320 & 0.97751 & 1.52200 & 167.549 & 127.831 & 180.0 \\
\hline$S_{16}$ & 2 & -111.9301 & $\mathrm{C}_{1}$ & 1.38218 & 1.13564 & 1.37162 & 69.991 & 120.612 & 19.955 \\
\hline$S_{17}^{*}$ & 1 & -111.9247 & $C_{1}$ & 1.31861 & 1.97155 & 2.08076 & 26.789 & 51.174 & 84.632 \\
\hline$S_{18}^{*}$ & 3 & -111.9242 & $\mathrm{C}_{1}$ & 1.30354 & 2.27990 & 1.02314 & 49.102 & 113.909 & 106.460 \\
\hline$S_{19}^{*}$ & 1 & -111.9005 & $\mathrm{C}_{s}$ & 1.24442 & 1.65359 & 1.65359 & 61.602 & 61.602 & 157.125 \\
\hline $\mathrm{S}_{20}^{*}$ & 6 & -111.8028 & $\mathrm{C}_{\infty v V}$ & 1.04821 & 2.24551 & 1.29467 & 179.145 & 0.314 & 180.129 \\
\hline $\mathrm{T}_{1}$ & 3 & -112.0122 & $\mathrm{C}_{2 v}$ & 1.77007 & 1.09488 & 1.64942 & 65.438 & 37.137 & 180.0 \\
\hline $\mathrm{T}_{2}$ & 3 & -112.0067 & $\mathrm{C}_{s}$ & 1.62111 & 1.29211 & 0.97555 & 44.034 & 171.845 & 0.0 \\
\hline$T_{3}$ & 3 & -111.9690 & $\mathrm{C}_{2 v}$ & 1.85668 & 1.99313 & 0.94603 & 28.165 & 83.960 & 180.0 \\
\hline $\mathrm{T}_{4}^{*}$ & 3 & -111.9498 & $\mathrm{C}_{s}$ & 2.46485 & 1.77115 & 0.93716 & 17.837 & 143.745 & 180.0 \\
\hline$T_{5}$ & 2 & -111.9264 & $\mathrm{C}_{s}$ & 1.47993 & 1.09729 & 1.28650 & 78.609 & 132.810 & 0.0 \\
\hline $\mathrm{T}_{6}^{*}$ & 1 & -111.8595 & $\mathrm{C}_{s}$ & 1.17135 & 2.08534 & 2.08534 & 59.685 & 59.685 & 107.771 \\
\hline $\mathrm{T}_{7}^{*}$ & 1 & -111.8584 & $\mathrm{C}_{1}$ & 1.29155 & 1.37052 & 2.26331 & 52.204 & 53.193 & 77.312 \\
\hline $\mathrm{T}_{8}^{*}$ & 3 & -111.8524 & $\mathrm{C}_{2 v}$ & 1.16570 & 2.60910 & 1.80592 & 36.259 & 121.297 & 180.0 \\
\hline $\mathrm{T}_{9}^{* d}$ & 4 & -111.8144 & $\mathrm{C}_{\infty v}$ & 1.00681 & 2.74973 & 1.61534 & 178.6 & 0.5 & 179.9 \\
\hline
\end{tabular}

${ }^{\mathrm{a}} \mathrm{M}$ : minimum; F: first index SP; S: second index SP; T: third index SP.

${ }^{\mathrm{b}}$ See Table II.

${ }^{\mathrm{c}} \mathrm{H}_{2}, \mathrm{CO}$ : dissociated structure, $r_{1}=r_{\mathrm{CO}}, r_{2}=r_{\mathrm{HH}}$.

${ }^{\mathrm{d}}$ Geometry not fully optimized because the linear geometry angle was an out of range error.

stretching of the $\mathrm{CH}$ bonds. We could trace the path from $\mathrm{M}_{1}$ to $\mathrm{F}_{2}$ by RGF starting in the direction $\theta$. But, $\mathrm{M}_{3}$ as starting point only gives the planar second index saddle $\mathrm{S}_{2}$, not $\mathrm{F}_{2}$. However, many different RGF curves connect $S_{2}$ and $F_{2}$. Note that the two structures (or three when considering the optical isomer of $\mathrm{F}_{2}$ ) collapse to one SP of index 1 at the MP2 level, as also indicated in ref. 20b.

Additionally, we found a linear saddle $\left(\mathrm{C}_{\infty v 0}\right)$ $\mathrm{HCOH}$, represented by $\mathrm{S}_{20}$, and a further $\mathrm{COH}_{2}$ structure in $\mathrm{S}_{12}$ of nonplanar $\mathrm{C}_{s}$ symmetry. A structure approaching linearity was also found for $\mathrm{CH}-\mathrm{HO}$, given as point $\mathrm{T}_{9}$ (SP of index 3 ), where the fourth eigenvalue is also near zero. The dis- tance between the two $\mathrm{H}$ atoms is $1.62 \AA$. The linear structure could not be calculated exactly using the GAMESS program.

A dissociation intermediate is the $\mathrm{C}_{2 v}$ minimum $\mathrm{CH}_{2} \cdots \mathrm{O}\left(\mathrm{M}_{7}\right)$ with the $\mathrm{C}-\mathrm{O}$ distance of $2.82 \AA$. At the very simple level of theory used, the fragment methylene $\left(\mathrm{CH}_{2}\right)$ does not come out in the proper equilibrium form, which is $\mathrm{a} \approx 135^{\circ}$ bending angle. ${ }^{31}$ (With the MP2 recalculation, the $\mathrm{M}_{7}$ structure disappears, and the dissociation to $\mathrm{CH}_{2}+\mathrm{O}$ does not have a van der Waals intermediate.)

If the hydrogens change to the oxygen moiety of the molecule, we find some structures along the pathway to $\mathrm{H}^{\delta-} \cdots \mathrm{COH}^{\delta+}$ that are not described 


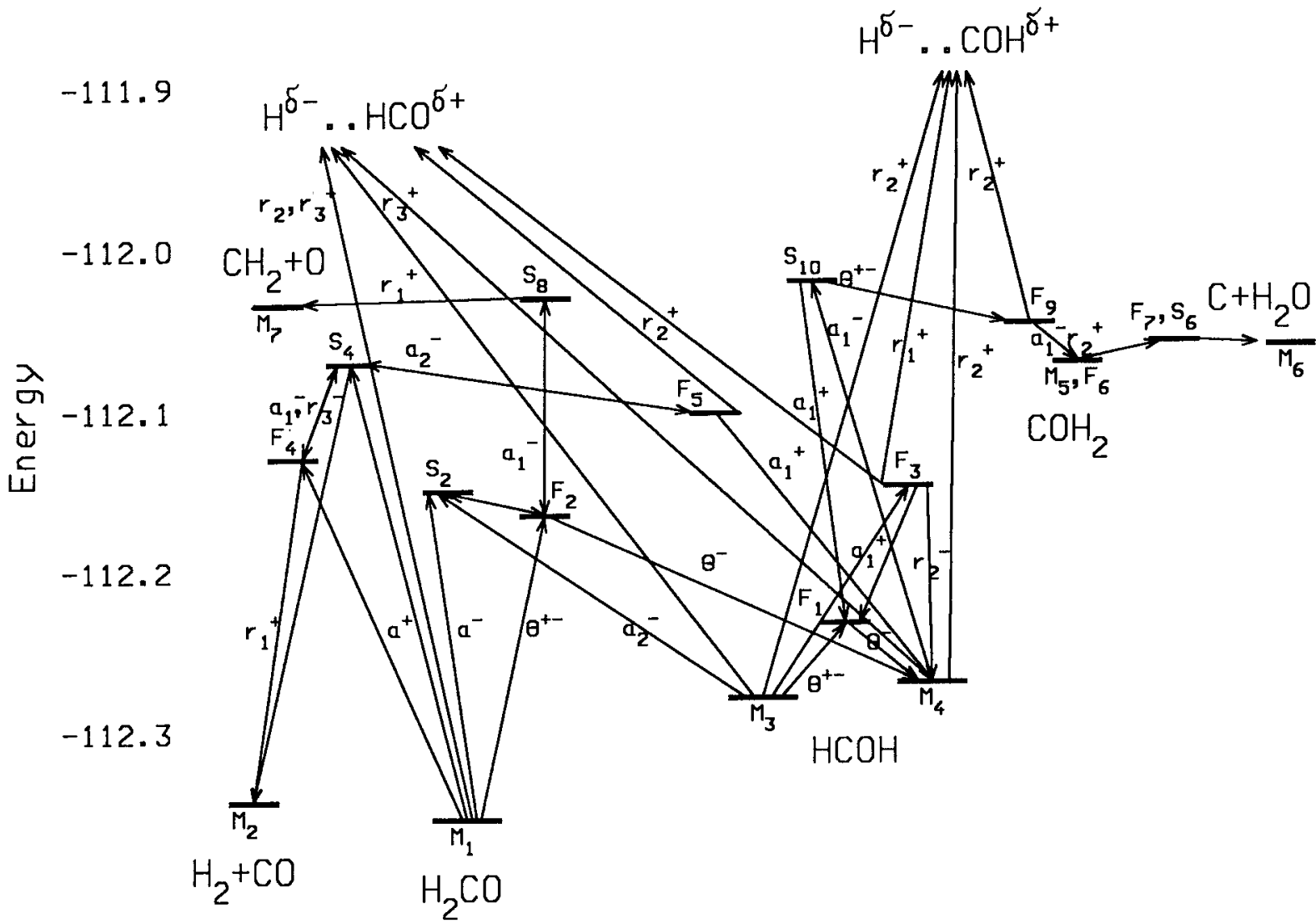

FIGURE 9. The RGF connections between the stationary points of the PES of $\mathrm{H}_{2} \mathrm{CO}$ described by arrows. $\mathrm{M}_{i}$ are the minima, $F_{i}$ are first index SPs, and $S_{i}$ are saddles of second index; "a" means angle $\alpha$, "+" means increase of the corresponding coordinate, "-" means decrease. The RHF or MP2 calculation does not appropriately describe the dissociation channel in the upper part of the figure, so we indicate it by $\mathrm{H}_{2} \mathrm{CO} \rightarrow \mathrm{H}^{\delta-} \cdots \mathrm{HCO}^{\delta+}$. There are many more RGF curves as shown in the scheme.

appropriately by a single reference RHF or MP2 wave function. We find $F_{10}$, two SPs of index 2, $\mathrm{S}_{17}, \mathrm{~S}_{18}$, and $\mathrm{T}_{7}$, all of $\mathrm{C}_{1}$ symmetry. There is a planar $\mathrm{C} \cdots \mathrm{H}_{2} \mathrm{O}$ minimum $\left(\mathrm{M}_{6}\right)$ with a $\mathrm{C}-\mathrm{H}$ van der Waals distance of $2.17 \AA$. $\mathrm{M}_{6}$ is also a minimum at the MP2 level. Further saddles are calculated: one of $\mathrm{C}_{2 v}$ symmetry at $\mathrm{F}_{7}$ and one of $\mathrm{C}_{1}$ symmetry at $\mathrm{F}_{8}$, a planar one at $\mathrm{S}_{5}$, a nonplanar $\mathrm{C}_{s}$ species at $S_{6}$, a linear one at $S_{14}$, and also one SP of planar structure $\mathrm{T}_{4}$ and one of $\mathrm{C}_{2 v}$ symmetry at $\mathrm{T}_{8}$. Finally, we obtain a set of structures leading to the decay into $\mathrm{H}^{\delta-} \cdots \mathrm{CO} \cdots \mathrm{H}^{\delta+}$ (generated by using RHF): the SP of $\mathrm{C}_{2 v}$ symmetry $\mathrm{F}_{12}$, a planar one at $\mathrm{S}_{19}$, and again an SP of $\mathrm{C}_{s}$ symmetry at $\mathrm{T}_{6}$ with index 3. A SP of planar structure is the decay product $\mathrm{H}_{2} \cdots \mathrm{C} \cdots \mathrm{O}$ as $\mathrm{F}_{13}$.

In the direction of decreasing bond lengths, there are usually many pathways leading to "clumped" atoms, which often diverge into high energy regions of the PES, or the SCF calculation diverges.
Such structures are of no use from the point of view of chemistry.

We can draw some general conclusions from the PES analysis of $\mathrm{H}_{2} \mathrm{CO}$ : The RGF pathway is-by definition-in general different from the IRC (intrinsic reaction coordinate) path, ${ }^{4}$ as well as from the GE (gradient extremal) path. ${ }^{22}$ For example, an IRC goes down from state $\mathrm{F}_{2}$ to $\mathrm{M}_{3}$. However, we could not find an adequate RGF curve along this line using the pure coordinate directions. We found $\mathrm{F}_{2}$ by some RGF curves starting in other StPs. However, RGF curves frequently give results similar to those of an IRC, or an "inverse IRC" search algorithm, ${ }^{33}$ but with better computational efficiency than the latter. In contrast, the direct search strategy for an SP of index 1 with GE path following is hindered by the emergence of turning points. $1,11,18,19 c, 21,22,33,34$ Therefore, in general, there is no GE connecting a minimum with a first index SP, even if both are connected by means of 
an IRC. GE pathways do not usually return to the SP search direction after passing a turning point. For RGF paths, the situation is much better. In most cases we even reach the next stationary point after passing a turning point. Handling of RGF pathways with bifurcation points has not been studied specifically in this chemical example. If a $\mathrm{BP}$ is crossed, either the algorithm does not react or it skips along a large Newton step of the corrector. If SCF convergence can be reached, the next accidentally found RGF curve behind the BP is followed.

\section{AZIDOTETRAZOLE ISOMERIZATION}

The unimolecular rearrangement between azidoazomethine and $1 \mathrm{H}$-tetrazole ${ }^{35}$ has been studied by RGF using the 4-31G basis set. The stationary points are reoptimized with $\mathrm{SCF} / 6-31+\mathrm{G}^{*}$ followed by a frequency analysis. The geometries of the stationary points are given in Table IV. Figure 10 shows the structures of the two minima and the $\mathrm{SP}$ in between. The ring opening of the $1 H$-tetrazole may be simulated by increasing the distance between the atoms $\mathrm{N}_{5}$ and $\mathrm{N}_{2}$. The corresponding RGF pathway is obtained by 14 predictor points with $S t L=0.3 \AA$ using eq. (14), and by 7 predictor points with $S t L=0.6 \AA$. No corrector step is called! A similar result is obtained using the DFP update of the Hessian: we need 12 and 8 predictor points, respectively, and do not need the corrector.

TABLE IV. Geometries $^{\mathrm{b}}$ of Azidoazomethine and $1 \mathrm{H}$-Tetrazole, and Intervening Saddle Point.

\begin{tabular}{lrrr}
\hline $6-31+\mathrm{G}^{* a}$ & $\begin{array}{c}\text { Azidoazo- } \\
\text { methine }\end{array}$ & Saddle point & 1 1H-tetrazole \\
\hline$E$ (a.u.) & -256.73032 & -256.55163 & -256.75408 \\
$r_{\mathrm{CN}_{2}}$ & 1.25105 & 1.27016 & 1.33020 \\
$r_{\mathrm{CN}_{3}}$ & 1.39599 & 1.38677 & 1.28925 \\
$r_{\mathrm{N}_{3} \mathrm{~N}_{4}}$ & 1.25555 & 1.32967 & 1.34141 \\
$r_{\mathrm{N}_{2} \mathrm{~N}_{5}}$ & 3.26697 & 2.03734 & 1.32606 \\
$r_{\mathrm{CH}_{6}}$ & 1.07852 & 1.06778 & 1.06776 \\
$r_{\mathrm{CH}_{7}}$ & 1.00203 & 0.99394 & 0.99409 \\
$\angle \mathrm{N}_{3} \mathrm{CN}_{2}$ & 124.149 & 117.999 & 108.178 \\
$\angle \mathrm{N}_{4} \mathrm{~N}_{3} \mathrm{C}$ & 112.292 & 104.583 & 105.756 \\
$\angle \mathrm{N}_{5} \mathrm{~N}_{2} \mathrm{C}$ & 75.400 & 95.826 & 108.012 \\
$\angle \mathrm{H}_{6} \mathrm{CN}_{2}$ & 126.025 & 127.837 & 125.023 \\
$\angle \mathrm{H}_{7} \mathrm{~N}_{2} \mathrm{C}$ & 111.636 & 124.231 & 131.226 \\
\hline
\end{tabular}

${ }^{\mathrm{a}} r$ in angstroms, angles in degrees.

${ }^{\mathrm{b}}$ All geometries have $\mathrm{C}_{s}$ symmetry.

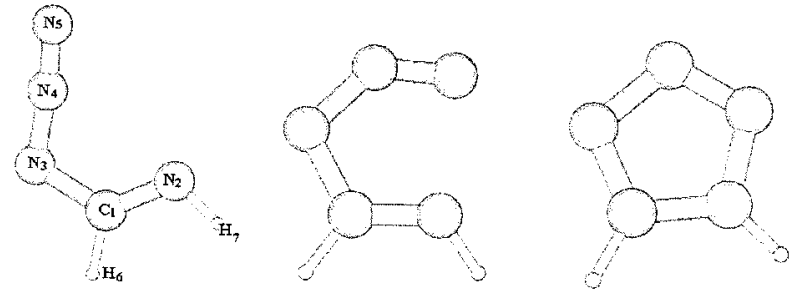

FIGURE 10. Minimum azidoazomethine, the transition structure, and minimum $1 H$-tetrazole at the RHF / 6-31+ $\mathrm{G}^{*}$ level.

In a second search for an out-of-plane SP, we use the out-of-plane distortion coordinate of $\mathrm{N}_{5}$ against the $\mathrm{N}_{2}$ as search direction. However, this approach finally leads back to the same planar SP given in Table IV. It demonstrates that there is a wide range of directions leading to the same SP. Of course, this RGF curve implies the passing through a turning point similar to the situation found in Figure 3. The steplength used in this test is $0.2 \mathrm{rad}$. Also, the reverse direction, starting at the SP and returning the out-of-plane search path, the RGF finds the pathway to tetrazole with $S t L=$ $0.3 \AA$.

With respect to azidoazomethine, the distance between the $\mathrm{N}_{5}$ and $\mathrm{N}_{2}$ atoms decreases to close the chain, and gives $1 \mathrm{H}$-tetrazole. This search direction also leads to the given SP. The tested predictor steplengths are 0.3 and $0.6 \AA$. The algorithm needs 25 and 12 predictor points, respectively, up to the SP. Hence, 2/0 corrector steps are called, respectively. Using the DFP update we need $27 / 14$ predictor steps up to the SP, and 1/0 corrector steps are called. The exact Hessian must be calculated at the stationary points only.

\section{Discussion}

The present investigation shows that a systematic reduced gradient following (RGF) allows for location of the stationary points of a PES. However, the method is not able to predict reliably which type of stationary point will be found. Usually, the RGF curves connect stationary points differing in their index by 1, as in Figure 2; however, this is only the rule if no BP is crossed. If an RGF curve bifurcates, the BP is a valley-ridge inflection point. The identification of a BP by rank deficit of the RGF matrix is straightforward in the case of symmetry of the PES, that is, if the distinguished coordinate follows the symmetry of the PES. Because the RGF method does not use the strategy of 
most of the other methods, namely to follow the minimum energy path, it forms a serious alternate method for the SP search. GE calculations still need derivatives of the Hessian. Hence, our results-for instance, on $\mathrm{H}_{2} \mathrm{CO}$ - are obtained with much less computational effort for the curve search. Because the $\mathrm{H}_{2} \mathrm{CO}$ rearrangements represents a six-dimensional problem, any global view on the PES is lost, and it seems impossible to be fully sure that the transition structures located are the only StPs. Although it is not practical to compute and fit a global PES for any but the smallest systems, it seems more possible to calculate a large number of one-dimensional RGF curves providing a dense network of curves that crosses the stationary points of the PES.

The similarity of the proposed algorithm with the old coordinate driving procedure (the distinguished coordinate method) is evident.9,10 Here, one coordinate is fixed, and all others are optimized with respect to the energy-similar to our corrector step. We recall that eqs. (12) were already formulated by Williams and Maggiora, ${ }^{10}$ but this explicit formula was not used in the sense of the tangent eq. (13). This is the reason why the distinguished coordinate method was not able to handle turning point problems. ${ }^{10,36}$ Müller ${ }^{29 a}$ provided an illustration of his model surface ${ }^{14}$ corresponding to that used in Figure 3 of this study. The pioneers of the distinguished coordinate method could not know that the discontinuities often observed in their pathways were connected with TP of the RGF curve. Let us take the two-dimensional case shown in Figure 3: The accentuated TP is the point of the RGF curve $E_{y}=0$ with the highest energy between the left minimum and the left SP. If we stop the minimization by the distinguished coordinate method at the TP, change the minimization of the energy into a maximization, and reverse the search direction, then we can reach the next SP. However, the path now runs downward near a ridge of the PES. This sequence of steps is, in general, executed by the RGF with the simple tangent predictor of eq. (13). It also illustrates the restricted possibilities of true reaction path following using the conventional distinguished coordinate method. By omitting the restriction to follow a RP we are able to find SPs on the side wall of the main valley. This case represents the general structural pattern on complex PES, cf. the left SP in Figure 3.

In summary, the predictor step of our method uses the tangent direction to the RGF curve, ${ }^{23}$ rather than a step along the distinguished coordinate itself. The proposed method has less to correct than the distinguished coordinate method. Thus, RGF avoid the "very rapid change of the optimized variable," which was one of the drawbacks of the old ansatz. ${ }^{37}$ RGF is not generally a method of following a reaction path; in fact, it even gains its power from the partial renunciation of this aim. But, the selection of one coordinate to follow the main stream of the reaction at the beginning of the search demands chemists intuition to reach a SP. This ensures favorable handling of the method. The mathematical line of reasoning, however, is based on the geometrical concept of RGF curves connecting (in an abstract way) the different stationary points, as well as valley-ridge inflection points. We have displayed a number of reduced gradient paths using two-dimensional test surfaces to show the properties of such curves. Finally, the stationary points of the $\mathrm{H}_{2} \mathrm{CO}$ potential energy surface were determined with the RHF method using the STO-3G basis for comparison with previous results. ${ }^{11}$ Another example demonstrating the efficiency of the method for a high-dimensional chemical system is the RHF/4-31G computation of the SP of the isomerization path from azidoazomethine to $1 H$-tetrazole. ${ }^{35}$

\section{Acknowledgments}

We thank Prof. J. Reinhold for stimulating our interest in Azido-Tetrazole isomerization. We are very grateful to Dr. Brenda P. Winnewisser for critically reading the manuscript, and also the referees for valuable suggestions and corrections.

\section{References}

1. D. Heidrich, W. Kliesch, and W. Quapp, Properties of Chemically Interesting Potential Energy Surfaces, Springer, Berlin, 1991.

2. A. Tachibana and K. Fukui, Theoret. Chim. Acta, 49, 321 (1978).

3. K. Laidler, Theory of Reaction Rates, McGraw-Hill, New York, 1969.

4. (a) K. Fukui, J. Phys. Chem., 74, 4161 (1970); (b) B. C. Garrett, M. J. Redmon, R. Steckler, D. G. Truhlar, K. K. Baldridge, D. Bartol, M. W. Schmidt, and M. S. Gordon, J. Phys. Chem., 92, 1476 (1988).

5. W. Quapp and D. Heidrich, Theoret. Chim. Acta, 66, 245 (1984).

6. D. Heidrich, In The Reaction Path in Chemistry: Current Approaches and Perspectives, D. Heidrich, Ed., Kluwer, Dordrecht, 1995, p. 1. 
7. (a) P. Y. Ayala and H. B. Schlegel, J. Chem. Phys., 107, 375 (1997); (b) A. Ulitzky and D. Shalloway, J. Chem. Phys., 106, 10099 (1997); (c) J. M. Anglada and J. M. Bofill, Int. J. Quant. Chem., 62, 153 (1997); (d) J. F. Rico, A. Aguado, and M. Paniagua, J. Mol. Struct. (Theochem), 371, 85 (1996); (e) Y. G. Khait, A. I. Panin, and A. S. Averyanov, Int. J. Quant. Chem., 54, 329 (1995); (f) C. Cárdenas-Lailhacar and M. C. Zerner, Int. J. Quant. Chem., 55, 429 (1995); (g) I. V. Ionova and E. A. Carter, J. Chem. Phys., 103, 5437 (1995); (h) A. Matro, D. L. Freeman, and J. D. Doll, J. Chem. Phys., 101, 10458 (1994); (i) J.-Q. Sun and K. Ruedenberg, J. Chem. Phys., 101, 2157 (1994); (j) Y. Abashkin and N. Russo, J. Chem. Phys., 100, 4477 (1994); (k) M. I. Ban, G. Dömötör, and L. L. Stacho, J. Mol. Struct. (Theochem), 311, 29 (1994); (1) P. L. A. Popelier, Chem. Phys. Lett., 228, 160 (1994); (m) S. F. Chekmarev, Chem. Phys. Lett., 227, 354 (1994); (n) J. Simons, Int. J. Quant. Chem., 48, 211 (1993); (o) C. J. Tsai and K. D. Jordan, J. Phys. Chem., 97, 11227 (1993); (p) S. Fischer and M. Karplus, Chem. Phys. Lett., 194, 252 (1992); (q) A. Banerjee and N. P. Adams, Int. J. Quant. Chem., 43, 855 (1992); (r) J. Nichols, H. Taylor, P. Schmidt, and J. Simons, J. Chem. Phys., 92, 340 (1990); (s) C. M. Smith, Int. J. Quant. Chem., 37, 773 (1990).

8. GAMESS_UK program: M. W. Schmidt, K. K. Baldridge, J. A. Boatz, S. T. Elbert, M. S. Gordon, J. H. Jensen, S. Koseki, N. Matsunaga, K. A. Nguyen, S. J. Su, T. L. Windus, M. Dupuis, and J. A. Montgomery, J. Comput. Chem., 14, 1347 (1993). (Internal mass-weighted coordinates have been used.)

9. (a) M. J. Rothmann and L. L. Lohr Jr., Chem. Phys. Lett., 70, 405 (1980); (b) P. Scharfenberg, Chem. Phys. Lett., 79, 115 (1981); (c) J. Comput. Chem., 3, 277 (1982).

10. I. H. Williams and G. M. Maggiora, J. Mol. Struct. (Theochem), 89, 365 (1982).

11. K. Bondensgård and F. Jensen, J. Chem. Phys., 104, 8025 (1996).

12. S. Wolfram, Mathematica (Version 2.2), 1993.

13. R. M. Minyaev, W. Quapp, G. Subramanian, P. v. R. Schleyer, and Y. Ho, J. Comput. Chem., 18, 1792 (1997).

14. K. Müller and L. D. Brown, Theoret. Chim. Acta, 53, 75 (1979).

15. C. Gonzales and H. B. Schlegel, J. Chem. Phys., 95, 5853 (1991).

16. B. Eckhardt, Physica D33, 89 (1988).

17. E. Neria, S. Fischer, and M. Karplus, J. Chem. Phys., 105, 1902 (1996).

18. W. Quapp, O. Imig, and D. Heidrich, In The Reaction Path in Chemistry: Current Approaches and Perspectives, D. Heidrich, Eds., Kluwer, Dordrecht, 1995, p. 137.

19. (a) J.-Q. Sun and K. Ruedenberg, J. Chem. Phys., 100, 5836 (1994); (b) H. B. Schlegel, J. Chem. Soc. Faraday Trans., 90, 1569 (1994); (c) W. Quapp, J. Chem. Soc., Faraday Trans., 90, 1607 (1994); (d) P. Valtazanos and K. Ruedenberg, Theoret. Chim. Acta, 69, 281 (1986); (e) H. Metiu, J. Ross, R. Silbey, and T. F. George, J. Chem. Phys., 61, 3200 (1974).

20. (a) T. Taketsuga and T. Hirano, J. Chem. Phys., 99, 9806 (1993); (b) J. Mol. Struct. (Theochem), 130, 169 (1994); (c) J. L. Liao, H. L. Wang, and H. W. Xin, Chinese Sci. Bull., 40, 566 (1995); (d) T. Taketsuga and M. S. Gordon, J. Chem. Phys., 103, 10042 (1995); (e) T. Yanai, T. Taketsuga, and T. Hirano, J. Chem. Phys., 107, 1137 (1997).

21. W. Quapp, Theoret. Chim. Acta, 75, 447 (1989).
22. (a) D. K. Hoffman, R. S. Nord, and K. Ruedenberg, Theoret. Chim. Acta, 69, 265 (1986); (b) P. Jørgensen, H. J. A. Jensen, and T. Helgaker, Theoret. Chim. Acta, 73, 55 (1988).

23. (a) E. L. Allgower and K. Georg, Numerical Continuation Methods-An Introduction, Springer, Berlin, 1990; (b) H. Schwetlick, In Computational Mathematics, Vol. 13, Banach Center, Warsaw, 1984, p. 623.

24. W. Quapp, In The Reaction Path in Chemistry: Current Approaches and Perspectives, D. Heidrich, Eds., Kluwer, Dordrecht, 1995, p. 95.

25. F. H. Branin, IBM J. Res. Devel., 504 (1972).

26. P. Deuflhard and H. Hohmann, Numerische Mathematik, de Gruyter, Berlin, 1991.

27. W. C. Davidon, Variable Metric Methods for Minimization (AEC R\&D Report ANL-5990), Argonne National Lab, Argonne, 1959.

28. R. Fletcher and M. J. D. Powell, Comput. J., 6, 163 (1963).

29. (a) K. Müller, Angew. Chem., 92, 1 (1980); (b) P. K. Pearson, H. F. Schaefer III, and U. Wahlgren, J. Chem. Phys., 62, 350 (1975); (c) K. Ishida, K. Morokuma, and A. Komornicki, J. Chem. Phys., 66, 2153 (1977); (d) J. N. Murell, S. Carter, and L. O. Halonen, J. Mol. Spectrosc., 93, 307 (1982); (e) S. C. Ross and P. R. Bunker, J. Mol. Spectrosc., 101, 199 (1983); (f) Z. Bačić, R. B. Gerber, and M. A. Ratner, J. Phys. Chem., 90, 3606 (1986); (g) M. Mladenovic and Z. Bačić, J. Chem. Phys., 93, 3039 (1990); (h) T. J. Lee, and A. P. Rendel, Chem. Phys. Lett., 177, 491 (1991); (i) B. S. Jursic, Chem. Phys. Lett., 256, 213 (1996).

30. (a) J. D. Goddard and H. F. Schaefer III, J. Chem. Phys., 70, 5117 (1979); (b) J. A. Pople, K. Raghavachari, M. J. Frisch, J. S. Binkley, and P. v. R. Schleyer, J. Am. Chem. Soc., 105, 6389 (1983).

31. (a) P. R. Bunker, P. Jensen, W. P. Kraemer, and R. Beardsworth, J. Chem. Phys., 85, 3724 (1986); (b) Y. Yamaguchi, H. F. Schaefer III, and G. Frenking, Molec. Phys., 82, 713 (1994).

32. (a) Z. Havlas, T. Kovař, and R. Zahradnik, J. Mol. Struct. (Theochem), 136, 239 (1986); (b) J. Baker and P. M. W. Gill, J. Comput. Chem., 9, 465 (1988); (c) G. E. Scuseria and H. F. Schaefer III, J. Chem. Phys., 90, 3629 (1989); (d) L. D. Deng, T. Ziegler, and L. Y. Fan, J. Chem. Phys., 99, 3823 (1993); (e) R. D. van Zee, M. F. Foltz, and C. B. Moore, J. Chem. Phys., 99, 1664 (1993); (f) Y. Yamaguchi, R. B. Remington, J. F. Gaw, H. F. Schaefer III, and G. Frenking, Chem. Phys., 180, 55 (1994); (g) Y. Abashkin, N. Russo, and M. Toscano, Theoret. Chim. Acta, 91, 179 (1995); (h) J. S. K. Yu and C. H. Yu, Chem. Phys. Lett., 271, 259 (1997).

33. W. Quapp, Chem. Phys. Lett., 253, 286 (1996).

34. F. Jensen, J. Chem. Phys., 102, 6706 (1995).

35. (a) L. A. Burke, J. Elguero, G. Leroy, and M. Sana, J. Am. Chem. Soc., 98, 1685 (1976); (b) M. W. Wong, R. LeungToung, and C. Wentrup, J. Am. Chem. Soc., 115, 2465 (1993).

36. (a) C. W. Bauschlicher, H. F. Schaefer III, and C. F. Bender, J. Am. Chem. Soc., 98, 1653 (1976); (b) M. J. Rothmann, L. L. Lohr Jr., C. S. Ewig, and J. R. van Wazer, In Potential Energy Surfaces and Dynamics Calculations D. G. Truhlar, Ed., Plenum Press, New York, 1981, p. 653; (c) U. Burkert and N. L. Allinger, J. Comput. Chem., 3, 40 (1982); (d) J. Cioslowski, A. P. Scott, and L. Radon, Mol. Phys., 91, 413 (1997).

37. T. A. Halgren and W. N. Lipscomb, Chem. Phys. Lett., 49, 225 (1977). 